\title{
Dosimetry Estimate and Initial Clinical Experience with 90Y-PSMA-617
}

\author{
Hendrik Rathke ${ }^{1}$, Paul Flechsig ${ }^{1}$, Walter Mier ${ }^{1}$, Marcus Bronzel ${ }^{2}$, Eleni Mavriopoulou ${ }^{1}$, Markus Hohenfellner ${ }^{3}$, \\ Frederik Lars Giesel ${ }^{1}$, Uwe Haberkorn ${ }^{1,4,5}$, and Clemens Kratochwil ${ }^{1}$ \\ ${ }^{I}$ Department of Nuclear Medicine, University Hospital Heidelberg, Heidelberg, Germany; ${ }^{2}$ ABX-CRO, Dresden, Germany; \\ ${ }^{3}$ Department of Urology, University Hospital Heidelberg, Heidelberg, Germany; ${ }^{4}$ Cooperation Unit Nuclear Medicine, German \\ Cancer Research Center, Heidelberg, Germany; and ${ }^{5}$ Translational Lung Research Center Heidelberg, German Center for Lung \\ Research, Heidelberg, Germany
}

Because of different physical properties, the $\beta$-emitters ${ }^{177} \mathrm{Lu}$ and ${ }^{90} \mathrm{Y}$ offer specific radiologic-biologic advantages in dedicated clinical situations. Our objective was to introduce ${ }^{90}$ Y-labeled prostatespecific membrane antigen (PSMA)-617 to clinical application, providing additional avenues for personalized medicine. Here, we present our dosimetry estimate for ${ }^{90} \mathrm{Y}-\mathrm{PSMA}-617$, report first clinical experiences, and discuss the advantages and drawbacks of varying the $\beta$-emitter in PSMA-targeting radioligand therapy. Methods: To approximate radiation dosimetry, 4 patients with metastatic castration-resistant prostate cancer underwent serially performed imaging up to 1 wk after ${ }^{177}$ Lu-PSMA-617 therapy. Time-activity curves were extrapolated to the half-life of ${ }^{90} \mathrm{Y}$, and OLINDA was used to calculate the dosimetry estimate. In clinical practice, 11 patients with PSMApositive lymph-nodal bulk disease were stratified to receive ${ }^{90} \mathrm{Y}$ PSMA-617 radioligand therapy (mean, 3.2 GBq; range, 2.8-3.7 $\mathrm{GBq}$ ); afterward, safety lab tests, prostate-specific antigen (PSA) response, and clinical findings were thoroughly followed. Results: The projected dosimetry for ${ }^{90} \mathrm{Y}-\mathrm{PSMA}-617$ estimated a mean kidney dose of $3.47 \pm 1.40 \mathrm{~Gy} / \mathrm{GBq}$, red marrow dose of $0.11 \pm 0.04 \mathrm{~Gy} / \mathrm{GBq}$, and salivary gland dose of $5.57 \pm 1.34 \mathrm{~Gy} / \mathrm{GBq}$; randomly chosen metastases were approximated with $22.8 \pm 16.10 \mathrm{~Gy} / \mathrm{GBq}$. The observed acute hematologic toxicity ( 5 cases of leukopenia and 2 of thrombocytopenia, all grade 1 or 2 ) and clinical side effects (2 cases of transient xerostomia and 1 of nausea, all grade 1 or 2 ), as well as PSA response (any PSA response, $7 / 11$ patients; $>50 \%$ PSA decline, 5/11 patients), were comparable to ${ }^{177}$ Lu-PSMA-617 literature data. Conclusion: A factor 3-4 lower treatment activity for ${ }^{90}$ Y-PSMA-617 translates into a comparable dosimetry estimate and clinical findings similar to those of ${ }^{177}$ Lu-PSMA-617. However, safety was demonstrated only for patients with oligometastatic disease. Further studies are needed to evaluate its potential in patients with more disseminated bone involvement or visceral metastasis.

Key Words: radionuclide therapy; PSMA-617; prostate cancer; $\mathrm{RLT}$; radiation dosimetry; yttrium-90

J Nucl Med 2019; 60:806-811

DOI: 10.2967/jnumed.118.218917

\footnotetext{
Received Aug. 7, 2018; revision accepted Oct. 22, 2018.

For correspondence or reprints contact: Hendrik Rathke, Department of Nuclear Medicine, University Hospital Heidelberg, Im Neuenheimer Feld 400, 69120 Heidelberg, Germany.

E-mail: hendrik.rathke@med.uni-heidelberg.de

Published online Nov. 2, 2018.

COPYRIGHT (C) 2019 by the Society of Nuclear Medicine and Molecular Imaging.
}

$\mathbf{F}$ irst clinical applications of prostate-specific membrane antigen (PSMA)-targeting radioimmunotherapy were based on antibody approaches with either ${ }^{90} \mathrm{Y}$ - or ${ }^{177}$ Lu-tagged J591 $(1,2)$. In this setting, ${ }^{177} \mathrm{Lu}$ was found preferable to ${ }^{90} \mathrm{Y}$. First, the slow pharmacokinetics of full-length antibodies provided an advantage to the longer half-life of ${ }^{177} \mathrm{Lu}(3)$. In addition, in patients with multiple bone metastases, the high-range $\beta$-particles of ${ }^{90} \mathrm{Y}$ delivered a higher radiation dose and, consequently, more hematologic toxicity, than was predicted with blood-based red-marrow dosimetry methods $(4,5)$. The better inherent imaging properties of ${ }^{177} \mathrm{Lu}$ (coemission of photons) were also a remarkable practical issue in the pre-PSMA PET/CT era.

The introduction of low-molecular-weight Glu-urea-based PSMA ligands accelerated tumor targeting in comparison to the former antibody-based approaches. The first generation of therapeutic PSMA ligands could be labeled only with ${ }^{131} \mathrm{I}(6,7)$. However, because of its conjugation to the DOTA chelator, the current reference compound PSMA-617 can be labeled with a variety of diagnostic or therapeutic radionuclides. Nevertheless, so far, considerable clinical experience is available only for ${ }^{177} \mathrm{Lu}-\mathrm{PSMA}-617$.

Also, the diagnostic clinical environment changed. PSMA PET/CT became more routinely available as an imaging surrogate to select only patients with PSMA-positive tumor phenotypes toward PSMA radioligand therapy (RLT).

On the basis of the physical characteristics of different therapeutic radionuclides, a theoretic rationale for patient stratification was applied. Most patients were treated with ${ }^{177}$ Lu-PSMA-617. Patients with diffuse-type red-marrow infiltration were selectively tailored toward $\alpha$-therapy with ${ }^{225}$ Ac-PSMA-617 to overcome the limitation of safety dosimetry based on the routinely applied blood model, which neglects bone metastases as a potentially relevant source for red marrow dose (8). Patients with bulky lesions but limited bone involvement were tailored toward ${ }^{90}$ Y-PSMA-617. In comparison to the low-energy ${ }^{177} \mathrm{Lu}(0.497 \mathrm{MeV})$, the $\beta$-particles emitted from ${ }^{90} \mathrm{Y}(2.28 \mathrm{MeV})$ have a higher penetration depth in water (mean range, $\sim 3 \mathrm{~mm}$ for ${ }^{90} \mathrm{Y}$ vs. $0.6 \mathrm{~mm}$ for ${ }^{177} \mathrm{Lu}$ ), and especially large lesions could benefit from increased cross-fire effects (9). This rationale has already been suggested for therapy of neuroendocrine tumors with radioactive somatostatin analogs (10); however, its clinical relevance has not yet been evaluated in a systematic, prospective manner.

The aim of this work was to approximate the radiation dosimetry of ${ }^{90}$ Y-PSMA-617 and to report clinical observations during the 
follow-up of the first patients receiving ${ }^{90}$ Y-PSMA-617. The treatment activities were widely dose-equivalent to ${ }^{177}$ Lu-PSMA-617 literature data.

\section{MATERIALS AND METHODS}

\section{Patients}

For the dosimetry approximation, preexisting data (11) were selected and reevaluated in an anonymized fashion without additional interventions involving human beings.

PSMA RLT was provided to patients with metastasized castrationresistant prostate cancer after exhausting (or being considered ineligible for) approved treatment options under the conditions of the updated article 37 of the Declaration of Helsinki, "Unproven Interventions in Clinical Practice." All patients gave written informed consent to receiving an experimental therapy. The retrospective data evaluation was approved by our institutional review board.

The appearance of a PSMA-positive tumor phenotype was assessed by PSMA PET/CT or PSMA SPECT (SPECT/CT) $(12,13)$. Patients were tailored to receive PSMA-617 tagged with either ${ }^{90} \mathrm{Y},{ }^{177} \mathrm{Lu}$, or ${ }^{225} \mathrm{Ac}$ on the basis of the respective physical nuclide characteristics to improve the theoretic absorbed dose distribution on the microdosimetry level (Fig. 1). Eleven patients with lymph-nodal bulk disease and fewer than 10 bone metastases were chosen for ${ }^{90}$ Y-PSMA RLT.

${ }^{90}$ Y-PSMA-617 was switched to ${ }^{177}$ Lu-PSMA-617 or ${ }^{225}$ Ac-PSMA617 after shrinkage of the lymph node bulk to below $2 \mathrm{~cm}$ in diameter or in cases of nonresponse.

\section{Preparation and Administration of Radiopharmaceuticals}

The PSMA-617 precursor was obtained by ABX and labeled in-house with ${ }^{90} \mathrm{YCl}_{3}$ (Yttriga; Eckert \& Ziegler Radiopharma $\mathrm{GmbH}$ ) using conditions identical to those for labeling with ${ }^{177} \mathrm{LuCl}_{3}(11)$. Quality control was assessed using reversed-phase high-performance liquid chromatography and instant thin-layer chromatography. The radiopharmaceutical was injected over $30 \mathrm{~s}$ through a low-protein-binding sterile filter (Filtropur S 0.2; Sarstedt). Twenty-four hours after application, bremsstrahlung scintigraphy was performed to validate physiologic biodistribution and positive tumor targeting and, further, to exclude paravasation. For dose reduction to the kidneys, patients received sufficient isotonic and isoosmolaric intravenous hydration (Sterofundin ISO; B. Braun) from $0.5 \mathrm{~h}$ before until $4 \mathrm{~h}$ after injection $(2,000 \mathrm{~mL}$ in total).

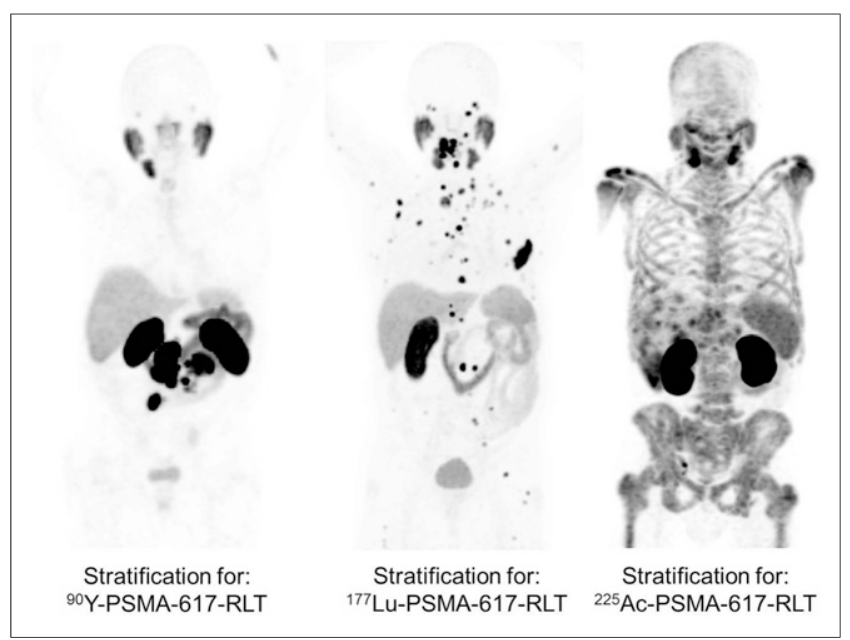

FIGURE 1. Bulky disease and stratification for ${ }^{90}$ Y-PSMA-617 RLT in comparison to patients eligible for ${ }^{177} \mathrm{Lu}$ - or ${ }^{225} \mathrm{Ac}-\mathrm{PSMA}-617 \mathrm{RLT}$. This concept is based on radiation-physical model assumptions but has not yet been prospectively proven in clinical setting.

\section{Dosimetry Approximation}

Blood samples were taken $30 \mathrm{~min}, 3 \mathrm{~h}, 20 \mathrm{~h}, 44 \mathrm{~h}$, and 4-8 d after therapeutic injection of ${ }^{177} \mathrm{Lu}$-PSMA-617. Urine was collected at intervals (1-4 h, 4-24 h, and 24-44 h) after injection. The activity in $1 \mathrm{~mL}$ of blood or urine was measured in a well counter (Bertold).

Planar imaging was performed $0.5 \mathrm{~h}, 3 \mathrm{~h}, 20 \mathrm{~h}, 44 \mathrm{~h}$, and 5-8 d after injection using a Hawkeye Millennium (GE Healthcare) with medium-energy whole-body collimation as previously described (11). Primary imaging was performed before voiding. Decay-corrected counts of the whole-body region of interest (ROI) (geometric mean) were assumed to correlate with the total injected dose. Dosimetry calculation and analysis were performed using QDOSE (ABXCRO). Organ-specific oval ROIs for lacrimal, parotid, and submandibular glands and freehand ROIs for liver, kidneys, spleen, bladder, and 6 tumor lesions were plotted. Organ ROIs were transferred to all other planar images. In cases of incomplete automatic coregistration, ROIs were adjusted manually. The whole-body remainder was calculated by subtracting all source organs from total body. Systemic redmarrow exposure was approximated using the blood-sampling-based model assumptions (8). Counts in the respective ROIs were translated to time-activity curves for further evaluation.

The activity of ${ }^{177} \mathrm{Lu}$-PSMA-617 at each imaging and blood-sampling time point was corrected for physical decay to the time point of injection, yielding the pharmacologic clearance. In a next step, the decay with the half-life of ${ }^{90} \mathrm{Y}$ was simulated. The new time-activity curves for total body, lacrimal and salivary glands, liver, spleen, kidneys, red marrow, and bladder were transferred to QDOSE, monoexponential curve fittings were performed, and the cumulated activity of each organ was integrated numerically (trapezoids) between measured time points and using the fit function from the last measured time point to infinity. The cumulated activity was divided through the injected activity to obtain residence times. Organ doses were calculated with OLINDA (HERMES Medical Solutions) using the adult male phantom; tumor lesions and salivary glands were determined using OLINDA's spheric modeling.

\section{Patient Course and Follow-up}

Lab tests, including white blood cells, red blood cells, platelets, urea, and creatinine, were checked and evaluated at baseline and every 2 wk by the local oncologist or in our department. Clinical examination and anamnesis were performed at admittance for each cycle. A calibrated radiometer (ThermoFisher Scientific FH40G-L10) was used to assess the dose rate $2 \mathrm{~m}$ from the patients at 4,24 , and $48 \mathrm{~h}$ after injection. Hematologic toxicity and adverse events were graded using version 5 of the Common Toxicity Criteria for Clinical Trials. Prostate-specific antigen (PSA) was checked every 4 wk. Imaging follow-up (PSMA PET/CT or PSMA SPECT/CT equivalent to baseline examination) was performed after 3 cycles or earlier whenever the treatment-concomitant bremsstrahlung scintigraphy implied morphologic changes and justified reassessment of lesion size (Fig. 2).

\section{RESULTS}

\section{Dosimetry}

The projected normal-organ dosimetry of ${ }^{90}$ Y-PSMA-617 (for the organs considered in the OLINDA adult male phantom) is presented in Table 1 . Because ${ }^{90} \mathrm{Y}$ is a pure $\beta$-emitting nuclide without long-distance radiation from source to nonsource organs, the whole-body remainder organs were almost equal and were summarized. Spheric model calculations revealed a dose of $5.57 \pm 1.34$ $\mathrm{Gy} / \mathrm{GBq}$ for salivary glands and $22.8 \pm 16.10 \mathrm{~Gy} / \mathrm{GBq}$ for metastases. The mean red marrow dose was $0.11 \pm 0.04 \mathrm{~Gy} / \mathrm{GBq}$, and the kidneys were $3.47 \pm 1.40 \mathrm{~Gy} / \mathrm{GBq}$. 


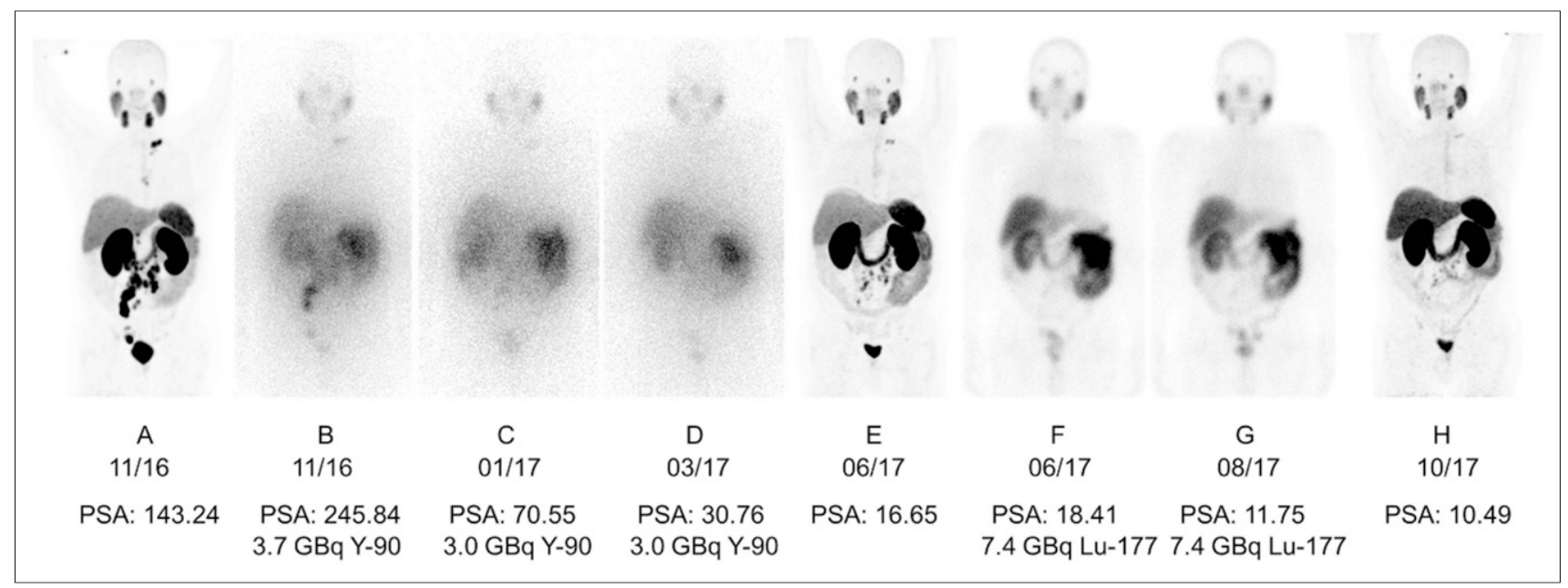

FIGURE 2. Patient with lymph-nodal bulk disease initially treated with ${ }^{90} \mathrm{Y}-\mathrm{PSMA}-617$. After shrinkage of bulk lesions, treatment was switched toward ${ }^{177} \mathrm{Lu}-\mathrm{PSMA}-617$. Staging examinations were performed with PSMA-11 PET/CT (A, E, and H), planar bremsstrahlung scans (B, C, and D), or scintigraphy of photo coemissions ( $F$ and $G$ ) during each treatment cycle.

Salivary glands, kidneys, and red marrow are considered to be dose-limiting organs for PSMA RLT. The calculated doses for the critical organs in comparison to ${ }^{225}$ Ac-PSMA-617, ${ }^{177} \mathrm{Lu}-\mathrm{PSMA}-$ 617, and ${ }^{131}$ I-MIP 1095 are presented in Table 2.

\section{Radiation Protection Issues}

Because of the urinary clearance of non-tumor-bound PSMA617 , in-patient administration for $48 \mathrm{~h}$ in our therapy ward was mandatory. Direct $\gamma$-emission was less than $1 \mu \mathrm{Sv} / \mathrm{h}$ per gigabecquerel at a 2-m distance from the patient at all time points.

\section{Side Effects}

Hematologic toxicity was observed over at least $8 \mathrm{wk}$ and after 3 cycles in total over 32 wk (Fig. 3). No grade 3 or 4 adverse events were observed. Two patients had grade 1 leukopenia under therapy. One patient had grade 2 leukopenia, and 2 patients had grade 2 leukopenia with preexisting grade 1 leukopenia before PSMA RLT; 1 patient had grade 1 thrombocytopenia and 1 patient grade 2 thrombocytopenia under therapy. Mean values for platelets, leukocytes, and creatinine are provided in Figure 3. Clinical

TABLE 1

Projected Organ-Absorbed Radiation Doses for 90Y-PSMA-617

\begin{tabular}{lcc}
\hline \multicolumn{1}{c}{ Organ } & Total dose (Gy/GBq) & SD \\
\hline Whole-body remainder organs & 0.11 & 0.04 \\
\hline Kidneys & 3.47 & 1.40 \\
\hline Liver & 0.50 & 0.14 \\
\hline Red marrow & 0.11 & 0.04 \\
\hline Osteogenic cells & 0.19 & 0.06 \\
\hline Spleen & 1.10 & 0.38 \\
Urinary bladder wall & 1.45 & 0.50 \\
\hline Total body & 0.14 & 0.04 \\
\hline Effective dose equivalent & 0.48 & 0.08 \\
Effective dose & 0.25 & 0.07 \\
\hline
\end{tabular}

side effects were transient xerostomia $(n=2)$ and nausea $(n=1)$. In sum, side effects under ${ }^{90}$ Y-PSMA-617 were comparable to the toxicity after an approximately 3-fold higher treatment activity of ${ }^{177}$ Lu-PSMA-617 RLT (14).

\section{PSA Response}

Eleven patients received 22 cycles of ${ }^{90}$ Y-PSMA-617, for a mean activity dose of $3.2 \pm 0.4 \mathrm{GBq}$ (range, 3.7-2.8 GBq). One patient requested an individual treatment regimen and postponed the second treatment cycle until the next PSA relapse; this exceptional case is presented in more detail in the next paragraph. The PSA follow-up for the regular treatment group is provided in Figure 4. Treatment was repeated after 2 mo (observation over $16 \mathrm{wk}$ ) in 7 patients and again after another 2 mo (observation over $24 \mathrm{wk}$ ) in 4 patients. To assess the therapeutic effect after the third cycle, clinical observation for ${ }^{90}$ Y-PSMA617 was performed over $32 \mathrm{wk}$ for those 4 patients. Two patients were restratified to short-range ${ }^{177}$ Lu-PSMA-617 or ${ }^{225}$ Ac-PSMA617 because of tumor shrinkage. Three patients were restratified to ${ }^{225}$ Ac-PSMA-617 because of an insufficient PSA response to ${ }^{90} \mathrm{Y}-$ PSMA-617. Three patients changed to another treatment regime (chemotherapy) because they were not willing to accept the risk of xerostomia from ${ }^{225}$ Ac-PSMA-617. Two patients paused therapy because of partial remission of tumor disease. In sum, 7 of 11 patients had any PSA response, and 5 of 11 patients had a PSA response of more than $50 \%$.

\section{Intraindividual Comparison of ${ }^{90}$ Y- Versus ${ }^{177}$ Lu-PSMA-617}

One patient received $3.7 \mathrm{GBq}$ of ${ }^{90}$ Y-PSMA-617 for the first cycle of PSMA RLT. The patient refused PSMA RLT repetitions every $8 \mathrm{wk}$, instead postponing retreatment until the next PSA relapse. Because of the response of the bulky lymph node metastases, the second cycle was tailored toward therapy with $8.5 \mathrm{GBq}$ of ${ }^{177} \mathrm{Lu}-\mathrm{PSMA}-617$ RLT. The course of this individual patient is presented in Figure 5. No relevant difference in the percentage decrease in PSA, time to PSA nadir, or other lab tests was observed between the ${ }^{90}$ Y-PSMA-617 RLT and the ${ }^{177}$ Lu-PSMA-617 RLT. Transient modest xerostomia was reported after both modalities, and temporary modest nausea was reported only after ${ }^{90}$ Y-PSMA617 ; however, nausea was no issue in the other ${ }^{90} \mathrm{Y}$ patients. 
TABLE 2

Dosimetry of Dose-Limiting Organs with Different PSMA Ligands and Nuclides

\begin{tabular}{|c|c|c|c|c|c|c|c|c|}
\hline \multirow[b]{2}{*}{$\begin{array}{l}\text { Dose-limiting } \\
\text { organ }\end{array}$} & \multicolumn{2}{|c|}{ This work } & \multicolumn{2}{|c|}{ Kratochwil et al. } & \multicolumn{2}{|c|}{ Zechmann et al. } & \multicolumn{2}{|c|}{ Kratochwil et al. } \\
\hline & $\begin{array}{c}90 \text { Y-PSMA-617 } \\
\text { (Gy/GBq) }\end{array}$ & $\begin{array}{c}90 \text { Y-PSMA-617 } \\
\text { (Gy/2.8 GBq) }\end{array}$ & $\begin{array}{c}{ }^{177} \text { Lu-PSMA-617 } \\
\text { (Gy/GBq) }\end{array}$ & $\begin{array}{c}\text { 177Lu-PSMA-617 } \\
\text { (Gy/7.4 GBq) }\end{array}$ & $\begin{array}{l}\text { 131/-MIP-1095 } \\
\text { (Gy/GBq) }\end{array}$ & $\begin{array}{c}\text { 131/-MIP-1095 } \\
\text { (Gy/3.7 GBq) }\end{array}$ & $\begin{array}{c}{ }^{225} \mathrm{Ac}-\mathrm{PSMA}-617 \\
\left(\mathrm{~S} \mathrm{v}_{\mathrm{RBE}} / \mathrm{MBq}\right)\end{array}$ & $\begin{array}{l}{ }^{225} \text { Ac-PSMA-617 } \\
\left(\mathrm{Sv}_{\mathrm{RBE} 5} / 7.4 \mathrm{MBq}\right)\end{array}$ \\
\hline Salivary glands & $5.57 \pm 1.34$ & 15.60 & 1.38 & 10.21 & 4.62 & 17.09 & 2.33 & 17.24 \\
\hline Kidneys & $3.47 \pm 1.40$ & 9.71 & 0.75 & 5.55 & 1.45 & 5.37 & 0.74 & 5.48 \\
\hline Red marrow & $0.11 \pm 0.04$ & 0.32 & 0.03 & 0.22 & 0.31 & 1.15 & 0.05 & 0.37 \\
\hline Metastases & $22.8(4.8-71.7)$ & 63.7 & $6.6(6-22)$ & 48.8 & $54.05(0-54.05)$ & & $5.7(1.3-9.8)$ & 42.1 \\
\hline
\end{tabular}

Data in parentheses are ranges.

\section{DISCUSSION}

The aim of this work was to introduce ${ }^{90}$ Y-PSMA-617 into clinical application to allow more personalized treatment regimens that reflect patients' individual situations.

In a first step, the radiation dosimetry for ${ }^{90}$ Y-PSMA-617 was approximated. We detected a treatment activity lower than that of ${ }^{177} \mathrm{Lu}-$ PSMA- 617 by a factor of 3-4, resulting in comparable radiation doses in organs at risk (Table 2). This result is well in line with the dosimetry estimates of ${ }^{90}$ Y-DOTATOC and ${ }^{177} \mathrm{Lu}$-DOTATATE, which have a blood and urine clearance highly similar to that of PSMA-617 $(15,16)$ and averaged a kidney dose converting factor of 3-3.5 (17). Treatment activities of up to $9.3 \mathrm{GBq}$ of ${ }^{177} \mathrm{Lu}$-PSMA-617 have previously been reported feasible (14), and thus, regarding safety issues, approximately a 3-GBq activity of ${ }^{90}$ Y-PSMA-617 per cycle was considered a dose-equivalent treatment activity. Our clinical findings with few observations of xerostomia and acute hematologic toxicities are well in line with the literature data for dose-equivalent ${ }^{177} \mathrm{Lu}-$ PSMA-617 $(14,18)$ and confirm the appropriateness of the anticipated standard treatment activity. At a first glance, relying on this constant factor, the average tumor-absorbed doses would be nearly doseequivalent. However, the investigator-defined assumption of uniform tumor uptake presents a critical simplification.

Balancing the respective advantages and drawbacks, it is mathematically possible to project a radionuclide's tumor-cell kill rate as a function of the underlying tumor size (19). According to this modeling, ${ }^{177} \mathrm{Lu}$ performs best for tumor diameters of $1.2-3.0 \mathrm{~mm}$, and the optimal lesion size to be treated with ${ }^{90} \mathrm{Y}$ is $28-42 \mathrm{~mm}$ (19). To close the gap between the recommended tumor sizes, a combination therapy with ${ }^{90} \mathrm{Y}$ - and ${ }^{177} \mathrm{Lu}$-radiopharmaceuticals has been modeled and validated preclinically (20). Later, a combination of

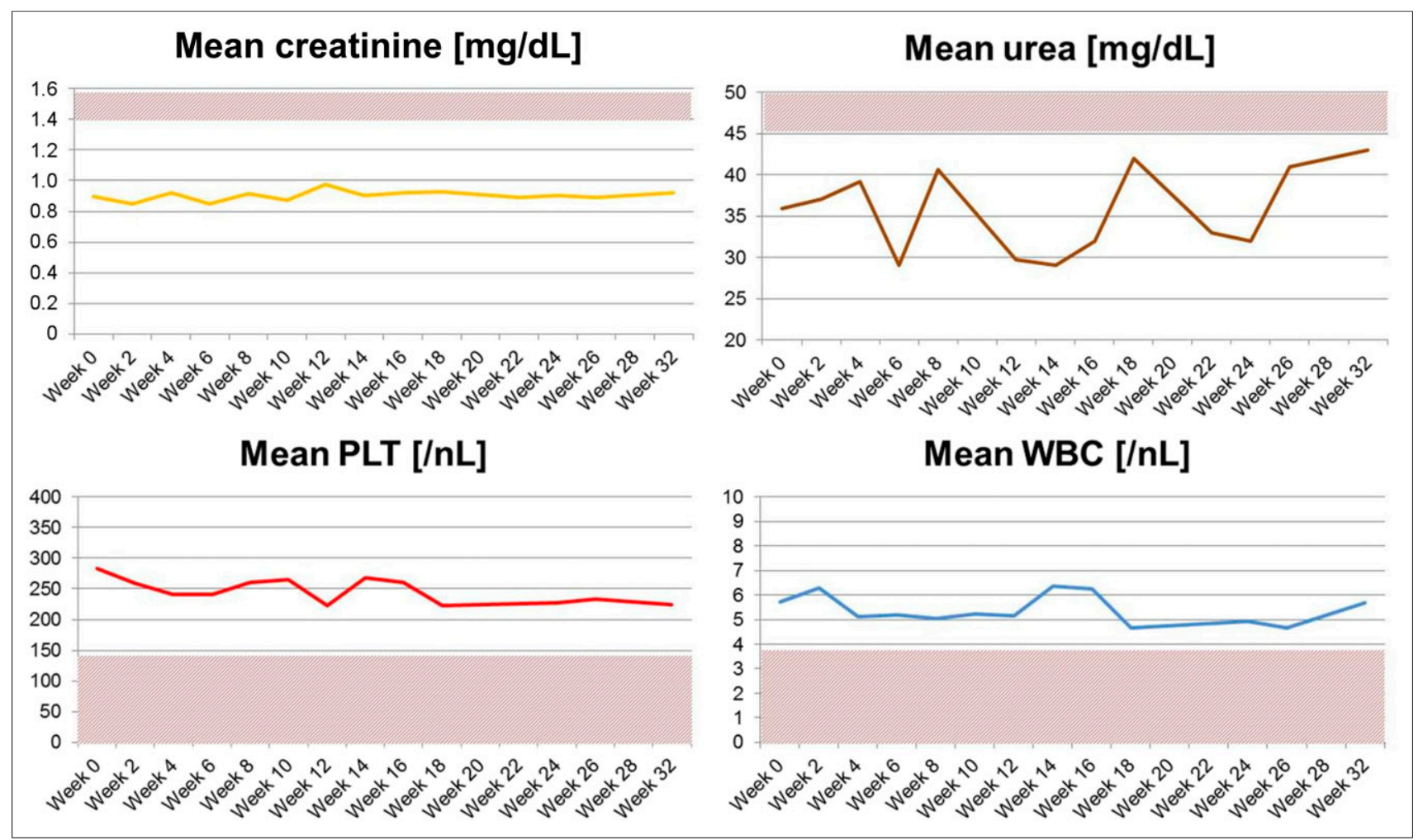

FIGURE 3. Lab curves of 10 patients under ${ }^{90} \mathrm{Y}-\mathrm{PSMA}-617 \mathrm{RLT}$ over maximum of 3 cycles. Therapeutic activities ranged from $3.7 \mathrm{GBq}$ over 3.0 $\mathrm{GBq}$ to our preferred treatment activity of $2.8 \mathrm{GBq}$ per cycle. Creatinine range $=0.5-1.4 \mathrm{mg} / \mathrm{dL}$; urea range $=<45 \mathrm{mg} / \mathrm{dL} ; \mathrm{platelet}(\mathrm{PLT}) \mathrm{range}=$ $150-440 / \mathrm{nL}$; white blood cell (WBC) range $=4-10 / \mathrm{nL}$. 


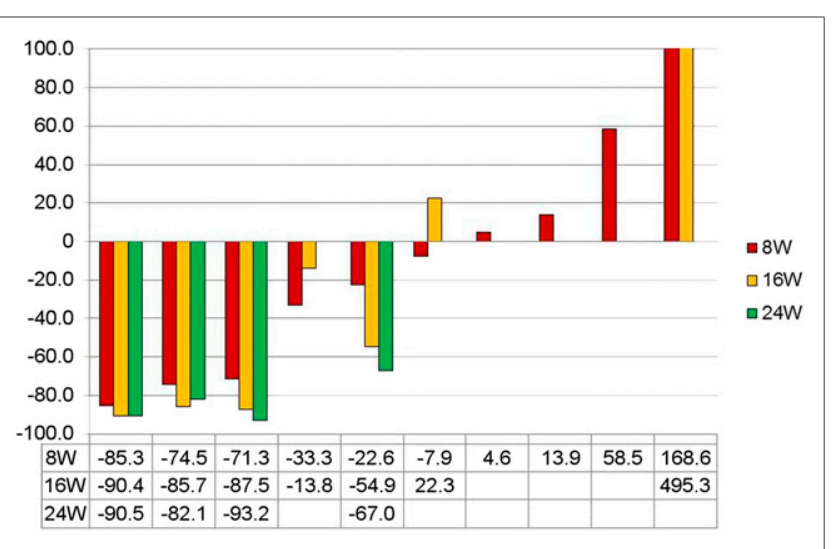

FIGURE 4. PSA to baseline (\%) for 10 patients eligible for ${ }^{90}$ Y-PSMA $\mathrm{RLT}$ at repeated 2-mo interval.

${ }^{90} \mathrm{Y} /{ }^{177} \mathrm{Lu}-\mathrm{DOTATATE}$ was used in therapy of neuroendocrine tumors (21), but no randomized comparison to the respective single nuclides has been performed yet.

Investigations of radiobiologic effects and physical theories over the past few decades support the thesis that PSMA RLT could potentially be refined by individually choosing the radiolabel nuclide. These might provide the most appropriate decay characteristics for the current clinical situation.

The cross-fire effect, as an effect of penetration of $\beta$-particles in tissue, is usually in the range of millimeters. Assuming a cell diameter of 10-20 $\mu \mathrm{m}$ and mean and maximum penetration ranges of 3 and $11 \mathrm{~mm}$, respectively, for ${ }^{90} \mathrm{Y}$ or 0.5 and $2 \mathrm{~mm}$, respectively, for ${ }^{177} \mathrm{Lu}$, the range might be up to several hundred cell diameters. This range leads to remarkable radiation dose deposits outside the originally targeted tumor cell. For small tumors, there is a major disadvantage because this energy does not contribute to the tumor-absorbed dose but is predominantly deposed in the surrounding normal tissue. Consequently, high-energy $\beta$-emitters such as ${ }^{90} \mathrm{Y}$ are limited in their potential to achieve cytotoxic dose levels in tiny tumors or micrometastases. On the other hand, wide-range $\beta$-particles can still deliver mentionable doses to target-negative cell clusters interspersed between tumor areas with sufficient inherent nuclide uptake. This ability may reflect an important advantage, especially as prostate cancer presents a

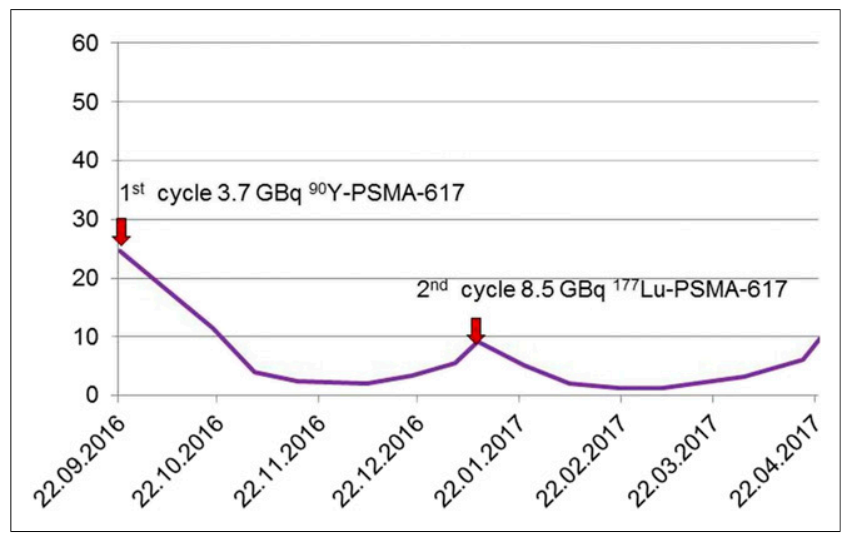

FIGURE 5. Lab curves of patient with initial stratification for ${ }^{90} \mathrm{Y}-$ PSMA-617 RLT. This patient requested individual treatment strategy and postponed second cycle until PSA progression. After initial therapeutic success, PSA relapsed. As diameter of tumor lymph nodes decreased, nuclide was changed to ${ }^{177}$ Lu-PSMA-617. pattern of high intratumoral heterogeneity on genetic, epigenetic, and phenotype levels $(22,23)$; in particular, bulky lesions may also contain hypoxic areas or areas of low perfusion.

On the basis of the respective considerations, only patients with oligometastatic bulky lesions were tailored to be treated with ${ }^{90} \mathrm{Y}-$ PSMA-617. Nuclide restratification was done if the average tumor size changed. Cocktail regimens were not applied.

However, in recent years it became evident that the release of proapoptotic factors from irradiated lesions can cause a response in closely related tumor cells, a phenomenon that is known as the radiation-induced bystander effect (24). Those cells are usually not, or only insufficiently, reached by the radiation itself. The presentation of tumor-specific molecules in combination with damage-associated molecular patterns can stimulate lymphocytes in the draining lymph nodes and cause immunologic antitumor activity even in distant metastases remote from the radiation zone (i.e., abscopal effect) (25). Consequently, the spill-out of radiation energy close to, but no longer inside, the tumor lesion, which for decades was considered off-target, has received growing attention since the introduction of cancer immunotherapy $(26,27)$. Radiation-induced inflammation of the surrounding lymphatic tissue may augment radiation-induced bystander or abscopal effects $(28,29)$; these observations encourage the use of high-energy $\beta$-emitters, especially in the field of lymph node metastasis.

Dose rate effects on tumors need to be mentioned, as lower dose rate and extended exposure time provide a greater time interval for DNA damage repair in tumor cells (30) and might lead to a preference for use of a nuclide with a short decay.

In addition to the absolute absorbed dose, the dose rate (depending on the nuclide half-life) and the homogeneity of the radiation zone (depending on tissue penetration range) may affect antitumor activity. Practical issues, such as regarding radiation protection or treatmentconcomitant imaging (both depending on coemission of photons), are important factors for appropriate handling in clinical routine. The physical characteristics of various medical isotopes suggested for therapeutic medical application in the field of PSMA RLT are summarized in Supplemental Table 1 (supplemental materials are available at http://jnm.snmjournals.org). ${ }^{90} \mathrm{Y}$ and ${ }^{177} \mathrm{Lu}$ present remarkable differences regarding their maximum tissue penetration range, half-life, and coemission of photons.

The practical advantage of ${ }^{90} \mathrm{Y}$ is a very low direct emission of radiation from patients, which is well in line with the literature value of its physical dose rate constant (point source in air) of approximately one sixth that of ${ }^{177} \mathrm{Lu}$ (Supplemental Table 1) $(31,32)$. Improving radiation safety is important with regard to the number of potential patients in an aging population. In return, bremsstrahlung imaging presents higher noise and lower resolution than nuclides with an inherent $\gamma$-coemission (Fig. 2). Theoretically, PET scanners could also be used for imaging the positron coemission from ${ }^{90} \mathrm{Y}$, but because of a very low ( $32 \mathrm{ppm})$ emission probability, this use is not widely applied in clinical routine $(33,34)$. Therefore, ${ }^{90} \mathrm{Y}$ PET/ CT will presumably not be considered for clinical routine in the near future. Vice versa, the main benefit of ${ }^{177} \mathrm{Lu}$ is its inherent imaging capabilities, which can achieve good resolution using a medium-energy collimator (Fig. 2). The photon emission probability of approximately $14 \%$ presents a superior trade-off between imaging quality and radiation protection issues in comparison to other medical $\beta$-emitters; for example, its $\gamma$-dose rate constant is lower by a factor of 10 than that of ${ }^{131}$ I but also than that of various copper isotopes, such as ${ }^{67} \mathrm{Cu}$ (Supplemental Table 1).

This work has some limitations, notably due to the limited number of patients under ${ }^{90}$ Y-PSMA-617 RLT. Therefore, straight 
recommendations cannot be provided. Regarding the dosimetry estimate, we assume lower actual absorbed doses, especially for the kidneys, because in our dosimetry model the intravenous hydration as above mentioned is not feasible. This limitation leads to probable lower kidney doses, as no grade 3 or 4 renal toxicity was observed over the $32 \mathrm{wk}$. However, a cohort of 11 patients does not cover all eventualities. Although the dose for the salivary glands was higher than in the ${ }^{177} \mathrm{Lu}$-PSMA-617 literature data, only 2 patients complained about transient xerostomia, and the long tissue range of ${ }^{90} \mathrm{Y}$ might not have such a high impact on the salivary glands, with its respective side effects.

\section{CONCLUSION}

${ }^{90}$ Y-PSMA-617, with a treatment activity lower by a factor of 3 than that of ${ }^{177}$ Lu-PSMA-617, is a safe option for selected patients with metastasized castration-resistant prostate cancer without extensive bone involvement. Further studies are necessary to evaluate the possible impact of disseminated bone metastasis on safety aspects. It remains questionable if the efforts of highly individualized patient tailoring are really warranted, because the response of our first patients was not remarkably different from that to previous treatments with standard activities of ${ }^{177} \mathrm{Lu}-\mathrm{PSMA}-617$. A cocktail combining ${ }^{177} \mathrm{Lu}$ - and ${ }^{90}$ Y-labeled PSMA ligands could be intriguing in patients with lesions of mixed size and should be evaluated in further studies. Considering radiation protection issues, ${ }^{90} \mathrm{Y}$ seems to provide some advantages to nursing staff, physicians, and caregiving relatives.

\section{DISCLOSURE}

Uwe Haberkorn, Clemens Kratochwil, and Walter Mier hold parts of a patent for PSMA-617. No other potential conflict of interest relevant to this article was reported.

\section{REFERENCES}

1. Milowsky MI, Nanus DM, Kostakoglu L, Vallabhajosula S, Goldsmith SJ, Bander NH. Phase I trial of yttrium-90-labeled anti-prostate-specific membrane antigen monoclonal antibody $\mathrm{J} 591$ for androgen-independent prostate cancer. $J$ Clin Oncol. 2004;22:2522-2531.

2. Bander NH, Milowsky MI, Nanus DM, Kostakoglu L, Vallabhajosula S, Goldsmith SJ. Phase I trial of ${ }^{177}$ lutetium-labeled J591, a monoclonal antibody to prostate-specific membrane antigen, in patients with androgen-independent prostate cancer. J Clin Oncol. 2005;23:4591-4601.

3. Vallabhajosula S, Kuji I, Hamacher KA, et al. Pharmacokinetics and biodistribution of ${ }^{111} \mathrm{In}$ - and ${ }^{177} \mathrm{Lu}$-labeled J591 antibody specific for prostate-specific membrane antigen: prediction of ${ }^{90} \mathrm{Y}-\mathrm{J} 591$ radiation dosimetry based on ${ }^{111} \mathrm{In}$ or ${ }^{177}$ Lu? J Nucl Med. 2005;46:634-641.

4. Vallabhajosula S, Goldsmith SJ, Hamacher KA, et al. Prediction of myelotoxicity based on bone marrow radiation-absorbed dose: radioimmunotherapy studies using ${ }^{90} \mathrm{Y}$ - and ${ }^{177} \mathrm{Lu}$-labeled J591 antibodies specific for prostate-specific membrane antigen. $J$ Nucl Med. 2005;46:850-858

5. Vallabhajosula S, Goldsmith SJ, Kostakoglu L, Milowsky MI, Nanus DM, Bander NH. Radioimmunotherapy of prostate cancer using ${ }^{90} \mathrm{Y}$ - and ${ }^{177} \mathrm{Lu}$-labeled J591 monoclonal antibodies: effect of multiple treatments on myelotoxicity. Clin Cancer Res. 2005;11:7195s-7200s.

6. Zechmann CM, Afshar-Oromieh A, Armor T, et al. Radiation dosimetry and first therapy results with a ${ }^{124} \mathrm{I} /{ }^{131} \mathrm{I}$-labeled small molecule (MIP-1095) targeting PSMA for prostate cancer therapy. Eur J Nucl Med Mol Imaging. 2014;41:1280-1292.

7. Afshar-Oromieh A, Haberkorn U, Zechmann C, et al. Repeated PSMA-targeting radioligand therapy of metastatic prostate cancer with ${ }^{131}$ I-MIP-1095. Eur J Nucl Med Mol Imaging. 2017;44:950-959.

8. Sgouros G. Bone marrow dosimetry for radioimmunotherapy: theoretical considerations. J Nucl Med. 1993;34:689-694.

9. Cremonesi M, Ferrari M, Di Dia A, et al. Recent issues on dosimetry and radiobiology for peptide receptor radionuclide therapy. $Q \mathrm{~J} \mathrm{Nucl} \mathrm{Med} \mathrm{Mol} \mathrm{Im-}$ aging. 2011;55:155-167.
10. Kunikowska J, Krolicki L, Hubalewska-Dydejczyk A, Mikolajczak R, SowaStaszczak A, Pawlak D. Clinical results of radionuclide therapy of neuroendocrine tumours with ${ }^{90} \mathrm{Y}$-DOTATATE and tandem ${ }^{90} \mathrm{Y} /{ }^{177} \mathrm{Lu}$-DOTATATE: which is a better therapy option? Eur J Nucl Med Mol Imaging. 2011;38:1788-1797.

11. Kratochwil C, Giesel FL, Stefanova M, et al. PSMA-targeted radionuclide therapy of metastatic castration-resistant prostate cancer with ${ }^{177} \mathrm{Lu}$-labeled PSMA617. J Nucl Med. 2016;57:1170-1176.

12. Rathke $\mathrm{H}$, Afshar-Oromieh A, Giesel FL, et al. Intraindividual comparison of ${ }^{99 \mathrm{~m} T c-m e t h y l e n e ~ d i p h o s p h o n a t e ~ a n d ~ p r o s t a t e-s p e c i f i c ~ m e m b r a n e ~ a n t i g e n ~ l i g a n d ~}$ ${ }^{99 \mathrm{~m}} \mathrm{Tc}-\mathrm{MIP}-1427$ in patients with osseous metastasized prostate cancer. $\mathrm{J} \mathrm{Nucl}$ Med. 2018;59:1373-1379.

13. Afshar-Oromieh A, Holland-Letz T, Giesel FL, et al. Diagnostic performance of ${ }^{68}$ Ga-PSMA-11 (HBED-CC) PET/CT in patients with recurrent prostate cancer: evaluation in 1007 patients. Eur J Nucl Med Mol Imaging. 2017;44:1258-1268.

14. Rathke H, Giesel FL, Flechsig P, et al. Repeated ${ }^{177}$ Lu-labeled PSMA-617 radioligand therapy using treatment activities of up to $9.3 \mathrm{GBq}$. J Nucl Med. 2018; 59:459-465.

15. Abuqbeitah M, Demir M, Uslu-Besli L, Yeyin N, Sonmezoglu K. Blood clearance and occupational exposure for ${ }^{177} \mathrm{Lu}$-DOTATATE compared to ${ }^{177} \mathrm{Lu}-$ PSMA radionuclide therapy. Radiat Environ Biophys. 2018;57:55-61.

16. Mair C, Warwitz B, Fink K, et al. Radiation exposure after ${ }^{177}$ Lu-DOTATATE and ${ }^{177}$ Lu-PSMA-617 therapy. Ann Nucl Med. 2018;32:499-502.

17. Cremonesi M, Botta F, Di Dia A, et al. Dosimetry for treatment with radiolabelled somatostatin analogues: a review. Q J Nucl Med Mol Imaging. 2010;54: 37-51.

18. Hofman MS, Violet J, Hicks RJ, et al. $\left[{ }^{177} \mathrm{Lu}\right]-P S M A-617$ radionuclide treatment in patients with metastatic castration-resistant prostate cancer (LuPSMA trial): a single-centre, single-arm, phase 2 study. Lancet Oncol. 2018;19:825-833.

19. O'Donoghue JA, Bardies M, Wheldon TE. Relationships between tumor size and curability for uniformly targeted therapy with beta-emitting radionuclides. $J$ Nucl Med. 1995;36:1902-1909.

20. de Jong M, Breeman WA, Valkema R, Bernard BF, Krenning EP. Combination radionuclide therapy using ${ }^{177} \mathrm{Lu}-$ and ${ }^{90} \mathrm{Y}$-labeled somatostatin analogs. $\mathrm{J} \mathrm{Nucl}$ Med. 2005;46(suppl 1):13S-17S.

21. Kunikowska J, Pawlak D, Bak MI, Kos-Kudla B, Mikolajczak R, Krolicki L. Long-term results and tolerability of tandem peptide receptor radionuclide therapy with ${ }^{90} \mathrm{Y} /{ }^{177} \mathrm{Lu}$-DOTATATE in neuroendocrine tumors with respect to the primary location: a 10-year study. Ann Nucl Med. 2017;31:347-356.

22. Massard C, Oulhen M, Le Moulec S, et al. Phenotypic and genetic heterogeneity of tumor tissue and circulating tumor cells in patients with metastatic castrationresistant prostate cancer: a report from the PETRUS prospective study. Oncotarget. 2016;7:55069-55082.

23. De Velasco MA, Uemura H. Prostate cancer immunotherapy: where are we and where are we going? Curr Opin Urol. 2018;28:15-24.

24. Sgouros G, Knox SJ, Joiner MC, Morgan WF, Kassis AI. MIRD continuing education: bystander and low dose-rate effects - are these relevant to radionuclide therapy? J Nucl Med. 2007;48:1683-1691.

25. Finkelstein SE, Timmerman R, McBride WH, et al. The confluence of stereotactic ablative radiotherapy and tumor immunology. Clin Dev Immunol. 2011;2011:439752.

26. Hansen AR, Massard C, Ott PA, et al. Pembrolizumab for advanced prostate adenocarcinoma: findings of the KEYNOTE-028 study. Ann Oncol. 2018;29: 1807-1813.

27. Formenti SC, Demaria S. Radiation therapy to convert the tumor into an in situ vaccine. Int J Radiat Oncol Biol Phys. 2012;84:879-880.

28. Moding EJ, Mowery YM, Kirsch DG. Opportunities for radiosensitization in the stereotactic body radiation therapy (SBRT) era. Cancer J. 2016;22:267-273.

29. Sharabi AB, Nirschl CJ, Kochel CM, et al. Stereotactic radiation therapy augments antigen-specific PD-1-mediated antitumor immune responses via crosspresentation of tumor antigen. Cancer Immunol Res. 2015;3:345-355.

30. Bernier J, Hall EJ, Giaccia A. Radiation oncology: a century of achievements. Nat Rev Cancer. 2004;4:737-747.

31. Meo SL, Cicoria G, Montini G, et al. Radiation emission dose from patients administered ${ }^{90}$ Y-labelled radiopharmaceuticals: comparison of experimental measurements versus Monte Carlo simulation. Nucl Med Commun. 2008;29: 1100-1105.

32. Olmstead C, Cruz K, Stodilka R, Zabel P, Wolfson R. Quantifying public radiation exposure related to lutetium-177 octreotate therapy for the development of a safe outpatient treatment protocol. Nucl Med Commun. 2015;36:129-134.

33. Carlier T, Willowson KP, Fourkal E, Bailey DL, Doss M, Conti M. ${ }^{90}$ Y-PET imaging: exploring limitations and accuracy under conditions of low counts and high random fraction. Med Phys. 2015;42:4295-4309.

34. Yue J, Mauxion T, Reyes DK, et al. Comparison of quantitative Y-90 SPECT and non-time-of-flight PET imaging in post-therapy radioembolization of liver cancer. Med Phys. 2016;43:5779. 